\title{
The effect of nonhomogeneous silver coating on the plasmonic absorption of Au-Ag core-shell nanorod
}

\author{
Jian Zhu $\cdot$ Fan Zhang $\cdot$ Jian-Jun Li $\cdot$ Jun-Wu Zhao
}

Published online: 4 October 2013

(C) The Author(s) 2013. This article is published with open access at SpringerLink.com

\begin{abstract}
Plasmonic light absorption properties of bimetallic $\mathrm{Au}-\mathrm{Ag}$ core-shell nanorod with nonhomogeneous Ag coating are investigated both theoretically and experimentally. When the Ag coating on the Au nanorod is not homogeneous, the overall aspect ratio and $\mathrm{Ag}$ composition greatly depends on the coating uniform, which strongly affects intensity changing and wavelength shift of the plasmonic absorption. When the transverse Ag coating is faster than longitudinal coating, the $\mathrm{Au}-\mathrm{Ag}$ core-shell nanorod could present two intense longitudinal plasmonic absorption peaks with equal intensity and small wavelength gap as the Ag shell thickness has a critical value. Furthermore, this critical Ag shell thickness could be decreased when the Au nanorod core has a small aspect ratio. When the longitudinal Ag coating is faster than transverse coating, the increasing intensity of longitudinal peak corresponding to outer $\mathrm{Ag}$ surface is always weaker than the longitudinal peak corresponding to $\mathrm{Au}-\mathrm{Ag}$ interface. Thus, we cannot obtain two equal intense plasmonic absorption peaks when the longitudinal Ag coating is thicker than the transverse coating. However, the transverse peak corresponding to $\mathrm{Au}-\mathrm{Ag}$ interface could be enhanced by decreasing the aspect ratio of the Au nanorod core. Thus, we can always find three distinct absorption peaks as the aspect ratio of $\mathrm{Au}$ nanorod is decreased and the longitudinal Ag coating is greater than transverse coating.
\end{abstract}

Keywords $\mathrm{Au}-\mathrm{Ag}$ bimetallic nanoparticles .

Nonhomogeneous silver coating · Core-shell structure .

Nanorod · Plasmonic absorption

\footnotetext{
J. Zhu $(\triangle) \cdot$ F. Zhang $\cdot$ J.-J. Li $\cdot$ J.-W. Zhao $(\triangle)$

The Key Laboratory of Biomedical Information Engineering of Ministry of Education, School of Life Science and Technology, Xi' an Jiaotong University, Xi'an, 710049, People's Republic of China e-mail: jianzhusummer@163.com

e-mail: nanoptzhao@163.com
}

\section{Introduction}

In recent years, noble metallic nanoparticles have received considerable attention owing to their novel optical properties based on localized surface plasmon resonance (LSPR). Although noble metal gold and silver have the same facecentered cubic crystal structure and similar lattice constants, silver nanoparticles display stronger LSPR absorption with higher plasmon energy (about $400 \mathrm{~nm}$ for Ag nanosphere and $520 \mathrm{~nm}$ for $\mathrm{Au}$ nanosphere) and more intense local electric field enhancement [1-3]. Therefore, Ag nanoparticles have been often used as substrates for surface-enhanced Raman scattering (SERS) [4] and sensing and imaging based on resonance light scattering $[5,6]$.

Because symmetry breaking induced redistribution of surface charges, the plasmonic optical properties are strongly dependent on particle shape. Thus, producing metallic nanoparticles with low symmetry (nonspherical symmetry) could improve plasmon tunability such as resonance wavelength and local field intensity [7, 8]. However, the synthesis of complex morphological silver nanoparticles such as $\mathrm{Ag}$ nanorod is much harder than gold [9]. One of the effective methods to achieve nanoparticles with well-defined morphology and silver surface is coating an $\mathrm{Ag}$ nanolayer on the premade gold nanoparticles with nonspherical symmetry [9-12].

By using the microwave-polyol method, $\mathrm{Au}-\mathrm{Ag}$ core-shell nanocrystals have been successfully synthesized [10]. The corresponding crystal structures and their growth mechanisms had also been studied. Furthermore, the transmission electron microscope (TEM) images observed by Tsuji et al. demonstrated that the shapes of initiated $\mathrm{Au}$ seeds greatly affect the shapes of formed $\mathrm{Ag}$ shells [13]. The $\mathrm{Au}-\mathrm{Ag}$ core-shell nanocubes with varying shaped cores have been reported by Gong et al. [14]. The prepared Au-Ag core-shell nanoparticles display very abundant and distinct LSPR 
characteristics, which are dependent on core shape and core size. By using iodide ions, the growth direction of the $\mathrm{Ag}$ shell on the gold nanodisk core could also be tuned, and then the shape of the coated Ag shell could also be controlled [15]. The LSPR and SERS response in $\mathrm{Au}$ dumb bells with silver coating have also been reported [9]; this type of core-shell $\mathrm{Au}-\mathrm{Ag}$ nanostructure is expected to serve as excellent SERS substrates because of the higher enhancement factors for silver as compared to gold. Ma et al. reported a facile method for generating $\mathrm{Au}-\mathrm{Ag}$ core-shell nanocubes with controllable edge lengths [16]. By varying the ratio of $\mathrm{Ag}$ ions to $\mathrm{Au}$ seeds, the thickness of the $\mathrm{Ag}$ shells could be finely tuned from 1.2 to $20 \mathrm{~nm}$, and the plasmon excitation of the $\mathrm{Au}$ cores would be completely screened when the Ag shell has a critical value of $3 \mathrm{~nm}$. The refractive index sensitivity of gold-silver core-shell nanoparticles has also been studied [17]. It has been found that coating a layer of silver brings about a higher refractive index sensitivity in comparison to the pure $\mathrm{Au}$ nanobars [18].

It is known that the $\mathrm{Ag}$ and $\mathrm{Au}$ nanorods have two LSPR bands corresponding to transverse and longitudinal resonance, respectively. The longitudinal plasmon bands are sensitive to the aspect ratio and could be tuned from visible to infrared region. An interesting topic arises from the effect of $\mathrm{Ag}$ coating on the LSPR of $\mathrm{Au}-\mathrm{Ag}$ core-shell nanorods. In our previous theoretical study, two transverse LSPR bands from outer $\mathrm{Ag}$ surface and $\mathrm{Au}-\mathrm{Ag}$ interface have been observed in $\mathrm{Au}-\mathrm{Ag}$ core-shell nanowires [19]. In the report of Yu et al. [20], the optical properties of bimetallic $\mathrm{Au}-\mathrm{Ag}$ core-shell nanorods were characterized by using a steady-state extinction spectra and ultrafast transient absorption spectroscopy. They have experimentally observed two longitudinal LSPR bands in $\mathrm{Au}-\mathrm{Ag}$ core-shell nanorods corresponding to the $\mathrm{Ag}$ shell and $\mathrm{Au}$ core. In the study of Liu et al., the silver coating induced blue shift, and enhancement of longitudinal plasmon mode was observed [21]. And the blue shift has been attributed to the changing of effective dielectric function and decreasing of the overall aspect ratio induced by silver coating.

In a recent report, it is interesting to find that the silver coating is not always homogeneous [3]. In the preparation method of Jiang et al., the thickness of the $\mathrm{Ag}$ shell at the side increases faster than that at the ends as the amounts of $\mathrm{AgNO}_{3}$ is increased [3]. How about the effect of nonhomogeneous silver coating on the plasmonic absorption properties of gold nanorod? In this report, we studied the plasmonic absorption properties of $\mathrm{Au}-\mathrm{Ag}$ core-shell nanostructure with nonhomogeneous Ag coating. It has been found that the anisotropy of $\mathrm{Ag}$ coating greatly affects intensity changing, wavelength shift, and peak number of the plasmonic absorption. Furthermore, the physical origin of the nonhomogeneous Ag coating-dependent LSPR tunability has also been discussed.

\section{The model}

The geometry of the core-shell structure $\mathrm{Au}-\mathrm{Ag}$ ellipsoidal nanorods with rotation symmetry is shown in Fig. 1. The Au ellipsoid core is modeled as a prolate (cigar shaped) spheroid, which is generated by rotating an ellipse about its major axis. Because of the rotation symmetry, the Au ellipsoid core has a semimajor axis $c_{1}$ and equal semiminor axis $a_{1}=b_{1}$. The outer surface of the $\mathrm{Ag}$ shell has semimajor axis $c_{2}$ and semiminor axis $a_{2}=b_{2}$. And the coated $\mathrm{Ag}$ shell has a transverse thickness of $t_{T}=a_{2}-a_{1}$ and longitudinal thickness of $t_{L}=c_{2}-c_{1}$. When the silver is homogeneously coated on the gold nanorod, $t_{T}$ and $t_{L}$ have the same value. In this model, the dielectric constant of the Au nanorod core, Ag coating shell, and environmental medium is given by $\varepsilon_{1}, \varepsilon_{2}$, and $\varepsilon_{3}$, respectively. It is important to note that the dielectric function $\varepsilon_{1}$ and $\varepsilon_{2}$ have real and imaginary wavelength $(\lambda)$-dependent components [22]. In our analysis, the size of the $\mathrm{Au}-\mathrm{Ag}$ nanostructure is much smaller than the incident wavelength. Thus, the nanoparticles are subjected to an almost uniform field and oscillate like a simple dipole. Therefore, the quasistatic approximation can be employed in the calculation. The basic equations of the polarizabilities along the principal axes and the plasmonic absorption cross-sections could be derived from the Laplace's equation and have been reported in [21, 23]. By plotting the absorption cross-sections with different wavelength, we could obtain the absorption spectrum.

\section{Results and discussion}

Absorption spectra of $\mathrm{Au}-\mathrm{Ag}$ nanorods with homogeneous silver coating

Figure 2a shows the calculated absorption spectra of bare $\mathrm{Au}$ nanorods and $\mathrm{Au}-\mathrm{Ag}$ core-shell nanorods with homogeneous $\mathrm{Ag}$ coating. In this calculation, the semiminor axis of the inner Au nanorod is set as $a_{1}=5 \mathrm{~nm}$, the aspect ratios of the $\mathrm{Au}$ nanorod is set as $p=c_{1} / a_{1}=4$, the environmental dielectric constant is set as $\varepsilon_{3}=1.0$. For pure Au nanorods, there are two LSPR absorption peaks corresponding to transverse and longitudinal resonance appearing at about 515 and $686 \mathrm{~nm}$, respectively. As the coating $\mathrm{Ag}$ shell thickness is increased from 1 to $11 \mathrm{~nm}$, both the transverse (denoted as peak 2) and longitudinal (denoted as peak 3) peaks blue shift and get intense. However, the shifting and intensity increase of the longitudinal peak are more intense, and then peak 2 has been merged gradually by peak 3 . What is more, the Ag coating results in a new plasmonic absorption peak taking place at shorter wavelength, which is denoted as peak 1 . As the coating $\mathrm{Ag}$ shell thickness is increased, the peak 1 red shifts slightly and gets intense greatly. All these Ag coating-dependent absorption properties are in good agreement with the 


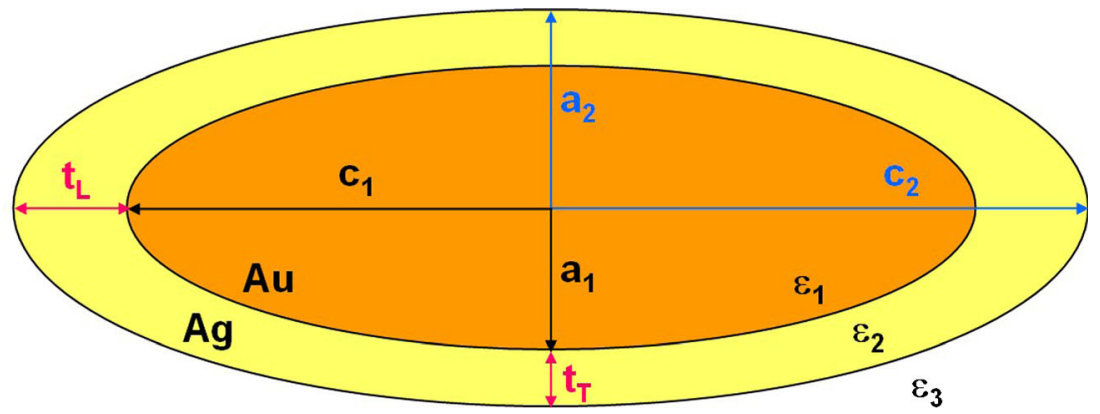

Fig. 1 Geometry of core-shell structure $\mathrm{Au}-\mathrm{Ag}$ ellipsoidal nanorods: $\varepsilon_{1}$, $\varepsilon_{2}, \varepsilon_{3}$ are the dielectric functions for the $\mathrm{Au}$ core, $\mathrm{Ag}$ nanoshell, and the semiminor axis. $t_{T}$ denotes the $\mathrm{Ag}$ shell thickness in transverse embedding regions respectively, $c$ denotes the semimajor axis and $a=b$

Fig. 2 a Calculated absorption spectra of $\mathrm{Au}-\mathrm{Ag}$ core-shell nanorods with homogeneous $\mathrm{Ag}$ coating, the Ag shell thickness is changed from 0 to $11 \mathrm{~nm}$. b Calculated absorption spectrum of $\mathrm{Au}-\mathrm{Ag}$ core-shell nanorods with nonhomogeneous Ag coating, $t_{T}=7.5 \mathrm{~nm}$ and $t_{L}=3.25 \mathrm{~nm}$
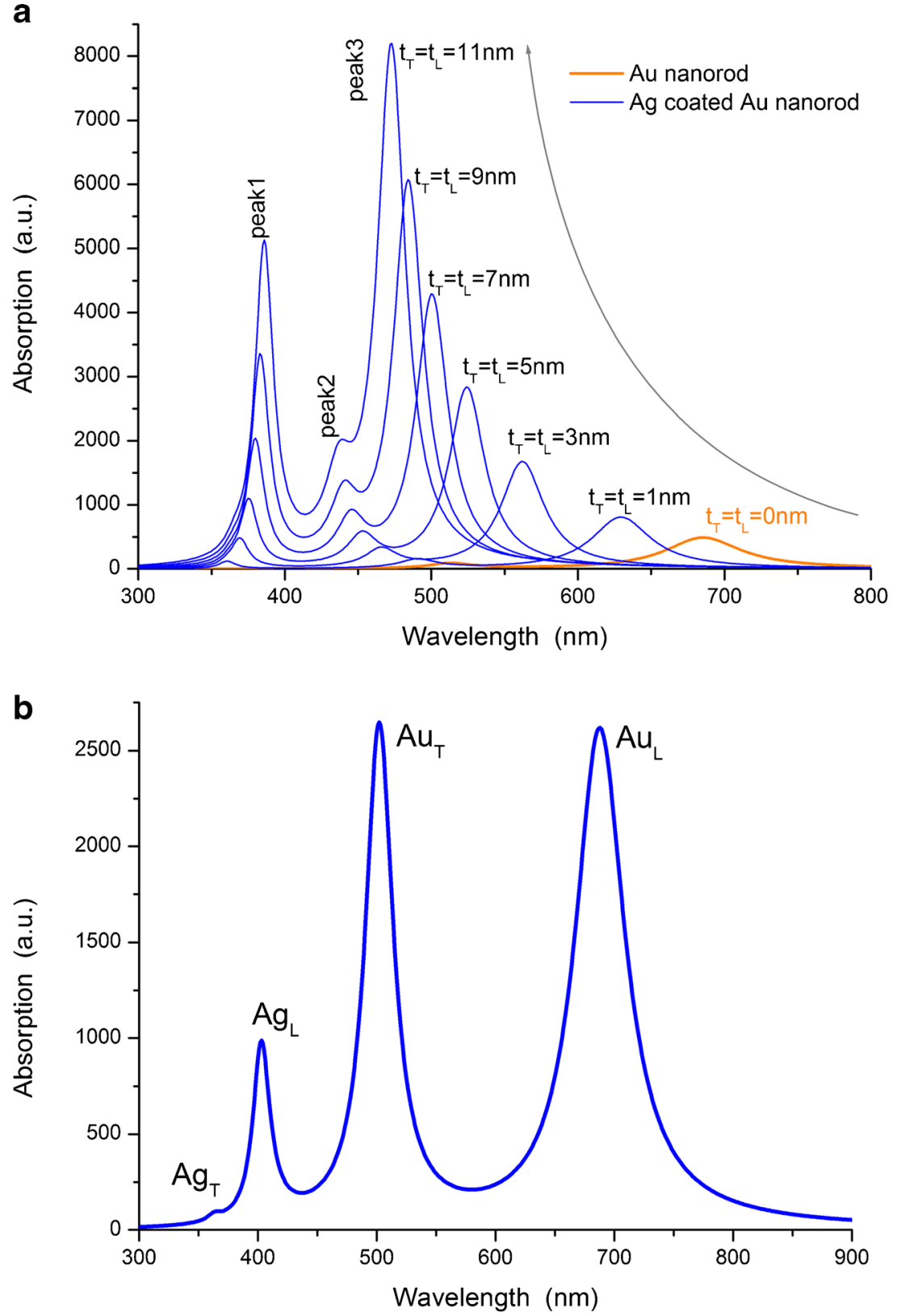
experimental results [21]. Because the Ag nanoparticles have more intense plasmonic absorption, the physical mechanism of the intensity increasing of peaks 2 and 3 could be resulted from the increase composition of $\mathrm{Ag}$ in the $\mathrm{Au}-\mathrm{Ag}$ bimetallic nanoparticles [21]. Because the Ag nanoparticles have shorter plasmon resonance wavelength, the intense blue shift of peaks 2 and 3 should also be attributed to the increase composition of $\mathrm{Ag}$ in the bimetallic nanoparticles. On the other hand, a homogeneous Ag layer coating lowers the overall aspect ratio of the core-shell nanostructure, which provides the minor reason of the blue shift of peak 3 .

Although the surface plasmons are coherent excitation and are affected by the coupling between metallic surfaces, different surface electron oscillations may take major effect on different plasmon modes. In our previous study of the transverse $\mathrm{LSPR}$ in $\mathrm{Au}-\mathrm{Ag}$ and $\mathrm{Ag}-\mathrm{Au}$ core-shell structure nanowires, the two plasmonic peaks have been assigned to outer surface of wall metal and the interface between core and wall metals, respectively [19]. Therefore, there should be four LSPR peaks in the $\mathrm{Au}-\mathrm{Ag}$ core-shell nanorod. However, one could only find three distinct plasmon peaks in Fig. 2a. The peaks 2 and 3 correspond to the transverse and longitudinal plasmon from $\mathrm{Au}-\mathrm{Ag}$ interface (denoted as $\mathrm{Au}_{\mathrm{T}}$ and $\mathrm{Au}_{\mathrm{L}}$ ). The peak 1 corresponds to the longitudinal plasmon from outer Ag surface (denoted as $\mathrm{Ag}_{\mathrm{L}}$ ). Because the transverse plasmonic absorption is always weaker than the longitudinal mode, the fourth peak corresponding to the transverse plasmon from outer $\mathrm{Ag}$ surface (denoted as $\mathrm{Ag}_{\mathrm{T}}$ ) is usually too weak to be observed and could only be found under certain conditions. For example, when the Ag coating is nonhomogeneous $\left(t_{T}=7.5 \mathrm{~nm}\right.$ and $\left.t_{L}=3.25 \mathrm{~nm}\right)$ and the environmental dielectric constant is increased to 3.5 , one can find four LSPR peaks in the absorption spectrum as shown in Fig. 2b. In the absorption spectral testing, the gold nanorods are usually suspended in the aqueous solution with a surrounding dielectric constant of $\varepsilon_{\text {surrounding }}=1.7$. Thus, it has been observed that the longitudinal absorption intensity is usually larger than the transverse intensity. In Fig. 2a, the surrounding dielectric constant is set as $\varepsilon_{3}=1.0$, and the longitudinal absorption intensity is also larger than the transverse intensity. However, when the surrounding dielectric constant is increased, the polarization of the dielectric media will affect the surface plasmon resonance. Because of the nonspherical symmetry, the effect on the transverse and longitudinal SPR is different. Previous experimental reports indicate that the transverse absorption increases and the longitudinal decreases when the surrounding dielectric constant is increased [24-26]. For example, by comparing the absorption spectra of bare Au nanorods and silica-coated Au nanorods, one can find that the transverse absorption increases and the longitudinal decreases as the silica coat thickness increased [24]. In Fig. 2b, in order to observe the fourth peak corresponding to the transverse plasmon from outer Ag surface, the surrounding dielectric constant is increased to $\varepsilon_{3}=3.5$. Therefore, the transverse plasmon absorption from $\mathrm{Au}-\mathrm{Ag}$ interface increases greatly, whereas the longitudinal plasmon absorption from $\mathrm{Au}-\mathrm{Ag}$ interface fades down.

Absorption spectra of $\mathrm{Au}-\mathrm{Ag}$ nanorods with nonhomogeneous silver coating

In Fig. 3, we studied the effect of nonhomogeneous $\mathrm{Ag}$ coating on the absorption spectral properties of $\mathrm{Au}-\mathrm{Ag}$ core-shell nanorods. For the case of $t_{T}=2 t_{L}$, i.e., the Ag shell coating in the transverse direction is faster than that of longitudinal direction, the overall aspect ratio decreases rapidly and the composition of $\mathrm{Ag}$ in the bimetallic particle increases rapidly. Therefore, the peak 3 blue shifts intensely and the peak 1 gets intense rapidly as the Ag shell thickness is increased. As shown in Fig. 3a, the intensity increase of peak 1 is faster than that of peak 3 , which is similar to the experimental observation [3]. Thus, the peak 1 at shorter wavelength becomes more intense than the peak 3 at longer wavelength when the Ag coating is thick, which is different from the calculation results of homogeneous coating case in Fig. 2a and the experimental result of [21]. Furthermore, because of this nonhomogeneous silver coating, the bimetallic nanostructures could present two intense plasmonic absorption peaks with equal intensity and small wavelength gap ca. $30 \mathrm{~nm}$ as the $\mathrm{Ag}$ shell thickness has a critical value, as shown in the inset of Fig. 3a. This plasmonic property provides potential for double-channel optical sensing based on plasmonic absorption [27]. In Fig. 3a, the disappearance of peak 2 in absorption spectrum is due to the following reasons. Firstly, the increase of outer Ag shell thickness leads to the great enhancement of peaks 1 and 3, which masks the weak transverse plasmon resonance of inner Au core. This trend could be clearly reflected in Fig. 2a. Secondly, the red shift of peak 1 and blue shift of peak 3 make the peak 2 merged together with peaks 1 and 3. Meanwhile, peak 2 only becomes distinct as the surrounding dielectric constant is large as shown in Fig. $2 b$. However, the surrounding dielectric constant in Fig. $3 \mathrm{a}$ is set as $\varepsilon_{3}=1$, which is much smaller than that of Fig. 2b. Therefore, the peak 2 in Fig. 3a is too weak to be observed.

In order to make the Ag coating-dependent intensity changing clearer, we also plotted the peak intensity of the absorption as the function of Ag coating thickness in Fig. 3c. When $t_{T}=$ $2 t_{L}$, the intensity of peak 3 is always a little greater than that of peak 1 as the $\mathrm{Ag}$ shell thickness is less than $8 \mathrm{~nm}$. The increasing speed of peak 3 fades down as the Ag coating is further increased. And then peaks 3 and 1 have the same intensity when $\mathrm{Ag}$ shell thickness reaches $9 \mathrm{~nm}$. However, the intensity difference between peaks 1 and 3 increases again when the $\mathrm{Ag}$ shell thickness is greater than $9 \mathrm{~nm}$. For the case of $t_{L}=2 t_{T}$, i.e., the Ag shell coating in the longitudinal direction is faster than that of transverse direction (but the ratio $t_{L} /$ 

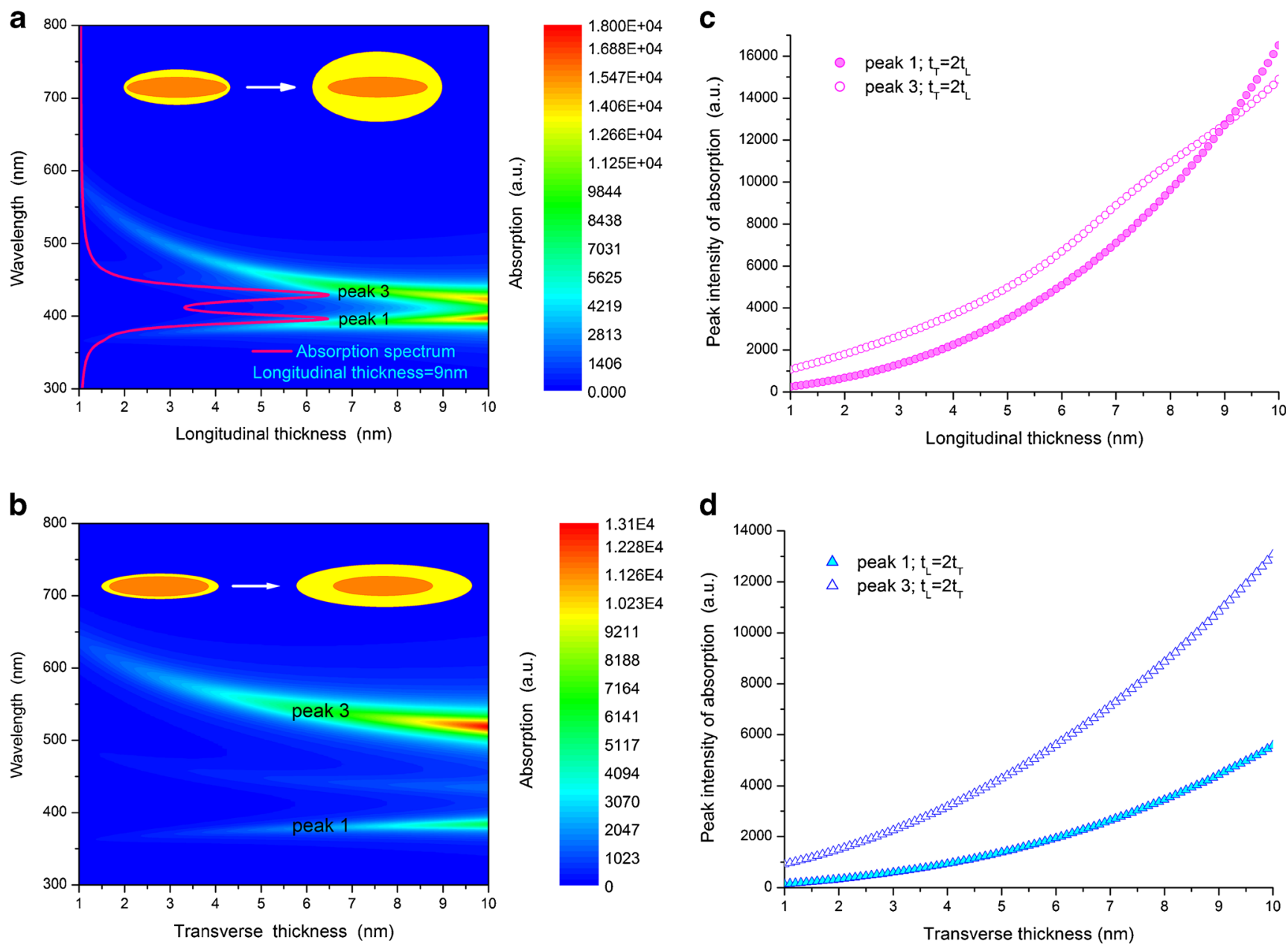

Fig. 3 Calculated absorption spectra of $\mathrm{Au}-\mathrm{Ag}$ core-shell nanorods with nonhomogeneous silver coating, the Au nanorod core has a large aspect ratio of $p=4$. a Transverse Ag shell thickness is larger, $t_{T}=2 t_{L}$. b Longitudinal Ag shell thickness is larger, $t_{L}=2 t_{T}$. c Peak intensity of

$t_{T}<p$ ), the overall aspect ratio decreases slowly and the $\mathrm{Ag}$ composition of the bimetallic particle also increases slowly. Therefore, the blue shift of peak 3 is gentle and the wavelength gap between peaks 1 and 3 is wide as shown in Fig. $3 b$. Furthermore, the intensity increase of peak 1 is also gentle and is always weaker than peak 3. As shown in Fig. 3c, d, the intensity difference between peaks 1 and 3 has been monotonously enlarged as the Ag coating is increased. And we cannot obtain two intense plasmonic absorption peaks with equal intensity when the longitudinal $\mathrm{Ag}$ coating is thicker as shown in Fig. 3d.

In order to find the effect of aspect ratio of the Au nanorod on the nonhomogeneous coating-dependent plasmonic absorption, we also plotted the absorption spectra of $\mathrm{Au}-\mathrm{Ag}$ core-shell nanorods when the aspect ratio of the Au nanorod core is decreased to $p=2$. As shown in Fig. 4, the way of the absorption intensity changing and wavelength shifting is similar to the cases of $p=4$. However, because of the decrease of the aspect ratio and the volume of the Au nanorod, the Ag

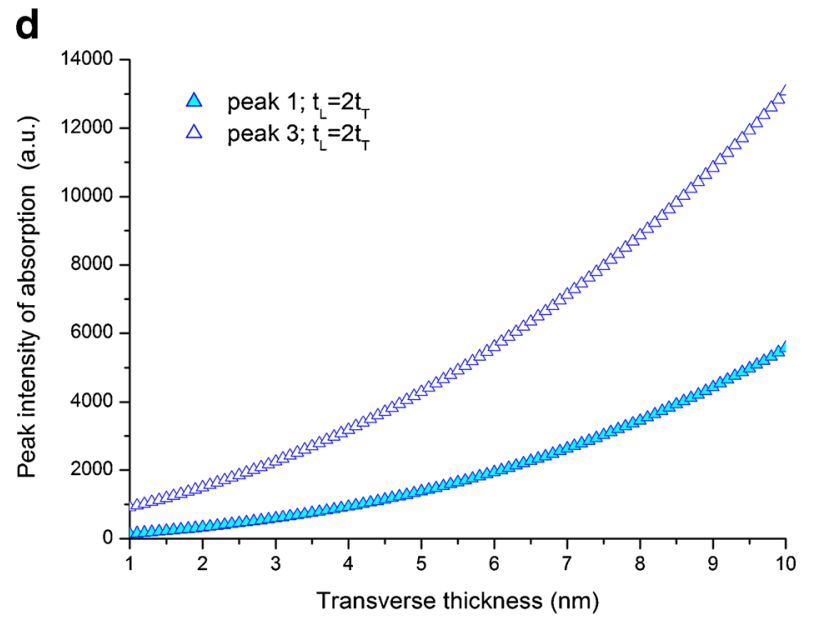

the absorption as a function of longitudinal Ag coating thickness. d Peak intensity of the absorption as a function of transverse Ag coating thickness, $\varepsilon_{3}=1.0$

coating-dependent Ag composition increase becomes intense. Therefore, the Ag coating-induced absorption intensity increase becomes rapid. Thus, we can obtain two intense plasmonic absorption peaks with equal intensity and small wavelength gap when the Ag coating thickness is thin. As shown in the inset of Fig. 4a, the $\mathrm{Au}-\mathrm{Ag}$ core-shell nanorods present two intense plasmonic absorption peaks with equal intensity and small wavelength gap ca. $45 \mathrm{~nm}$ as the longitudinal Ag shell thickness is $4 \mathrm{~nm}$. The peak intensity of the absorption as the function of longitudinal Ag coating thickness has been plotted in Fig. $4 \mathrm{c}$. When $t_{T}=2 t_{L}$, the intensity of peak 3 is always greater than that of peak 1 as the Ag shell thickness is less than $4 \mathrm{~nm}$. However, the increasing speed of peak 3 gets intense first and then fades down as the Ag coating is increased. At last, peaks 3 and 1 have the same intensity when $\mathrm{Ag}$ shell thickness reaches $4 \mathrm{~nm}$. In Fig. 4b, because the intensity discrepancy between transverse and longitudinal plasmonic absorption is decreased, the relative intensity of peak 2 is much greater than that of the absorption spectra in 

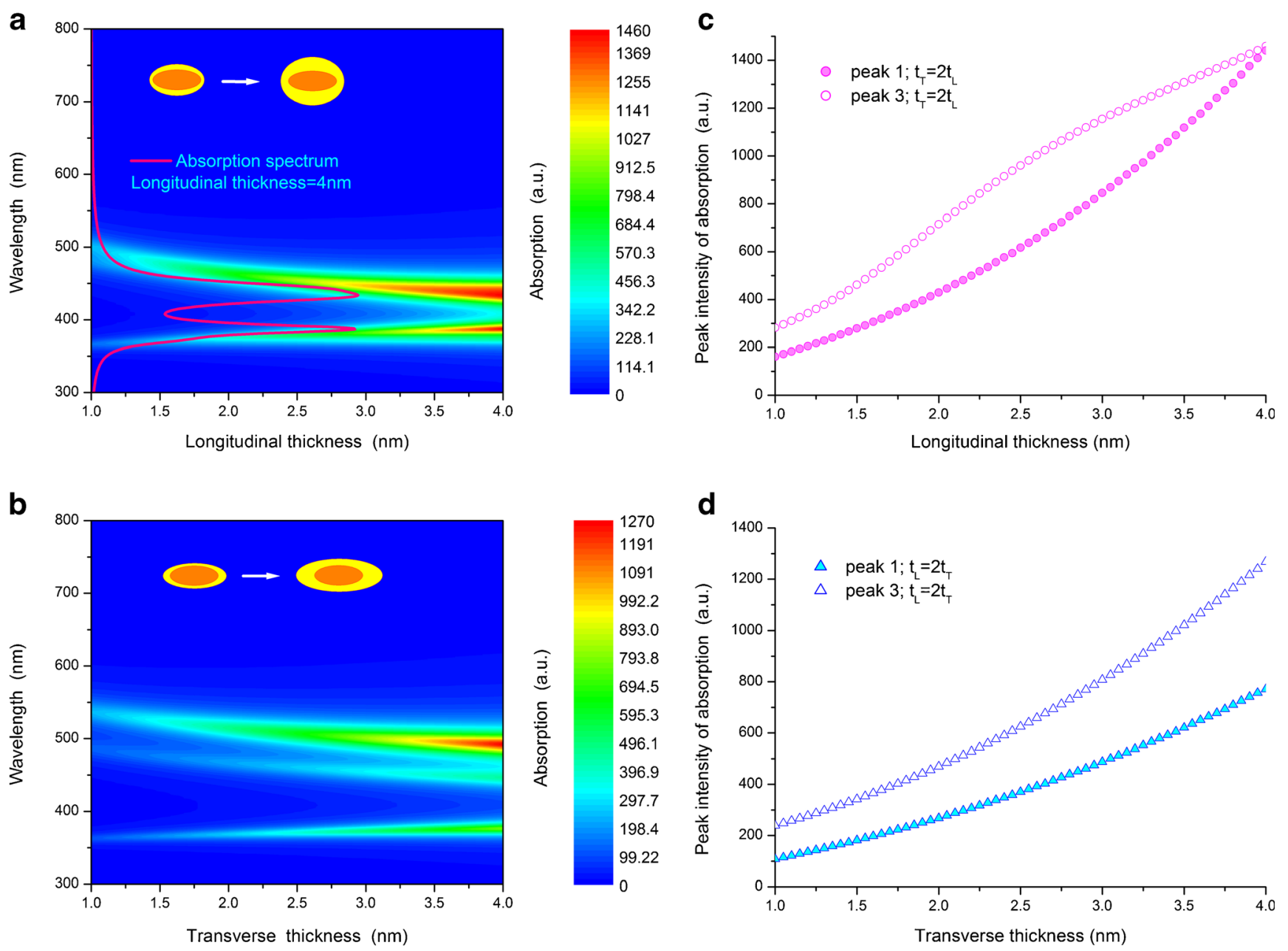

Fig. 4 Calculated absorption spectra of $\mathrm{Au}-\mathrm{Ag}$ core-shell nanorods with nonhomogeneous silver coating, the Au nanorod core has a small aspect ratio of $p=2$. a Transverse $\mathrm{Ag}$ shell thickness is larger, $t_{T}=2 t_{L}$. b Longitudinal Ag shell thickness is larger, $t_{L}=2 t_{T}$. c Peak intensity of

Fig. 3b. We can always find three distinct absorption peaks as the Au nanorod is short and the longitudinal Ag coating is thicker.

Experimental study of the absorption spectra of $\mathrm{Au}-\mathrm{Ag}$ nanorods with silver coating (transverse coating is larger than longitudinal coating)

\section{The preparation of Au nanorods}

Au nanorods were prepared according to a seed-mediated growth protocol developed by Sau and Murphy with some slight modification [28]. Initially, Au seed solution was prepared by quickly injecting $\mathrm{NaBH}_{4}(0.01 \mathrm{M}, 0.6 \mathrm{~mL})$ into the mixture of $\mathrm{HAuCl}_{4}(0.01 \mathrm{M}, 0.25 \mathrm{~mL})$ and CTAB $(0.1 \mathrm{M}$, $7.5 \mathrm{~mL}$ ) solution in a test tube of $15 \mathrm{~mL}$, followed by $1 \mathrm{~min}$ of violent stirring. The color of the solution changed from yellow to brownish yellow, which indicates the start formation of the $\mathrm{Au}$ seed. Then, the resultant solution was kept at

the absorption as a function of longitudinal Ag coating thickness. d Peak intensity of the absorption as a function of transverse Ag coating thickness, $\varepsilon_{3}=1.0$

$27{ }^{\circ} \mathrm{C}$ for $2 \mathrm{~h}$ for the next use. Secondly, to grow $\mathrm{Au}$ nanorods, $0.01 \mathrm{M} \mathrm{HAuCl}_{4}(4 \mathrm{~mL}), 0.01 \mathrm{M} \mathrm{AgNO}_{3}, 0.1 \mathrm{M}$ $\mathrm{AA}(0.64 \mathrm{~mL})$, and $1 \mathrm{M} \mathrm{HCl}$ were injected into $0.1 \mathrm{M}$ CTAB $(90 \mathrm{~mL})$ in order. With gentle stirring, the color of the solution changed from brownish yellow to transparent. Different aspect ratios of gold nanorods could be obtained by tuning the volume of $\mathrm{AgNO}_{3}$ and $\mathrm{HCl}$. Finally, $0.2 \mathrm{~mL}$ $\mathrm{Au}$ seed solution was injected to initiate growth reaction. Then the ultimate solution was kept at a constant temperature of $27{ }^{\circ} \mathrm{C}$ undisturbed overnight. During this time, the transparent solution progressively changed to purple or deep blue, depending on the aspect ratio of the $\mathrm{Au}$ nanorods. When the volume of $\mathrm{AgNO}_{3}$ is relatively small, we obtained the shorter $\mathrm{Au}$ nanorods with an aspect ratio of 3.3. Figure 5a depicted the synthesized shorter Au nanorods with an average length of about $40 \mathrm{~nm}$ and width of about $12 \mathrm{~nm}$. In order to get longer $\mathrm{Au}$ nanorods, the $\mathrm{AgNO}_{3}$ volume has been increased and $\mathrm{HCl}$ was added at the same time. As shown in Fig. 5b, longer Au nanorods with an aspect ratio 
Fig. 5 TEM images of a short Au nanorods with an aspect ratio of 3.3. b Long Au nanorods with an aspect ratio of 4.8. $\mathbf{c ~} \mathrm{Au}-\mathrm{Ag}$ core-shell nanorod with thin $\mathrm{Ag}$ shell thickness. d Au-Ag coreshell nanorod with thick Ag shell thickness
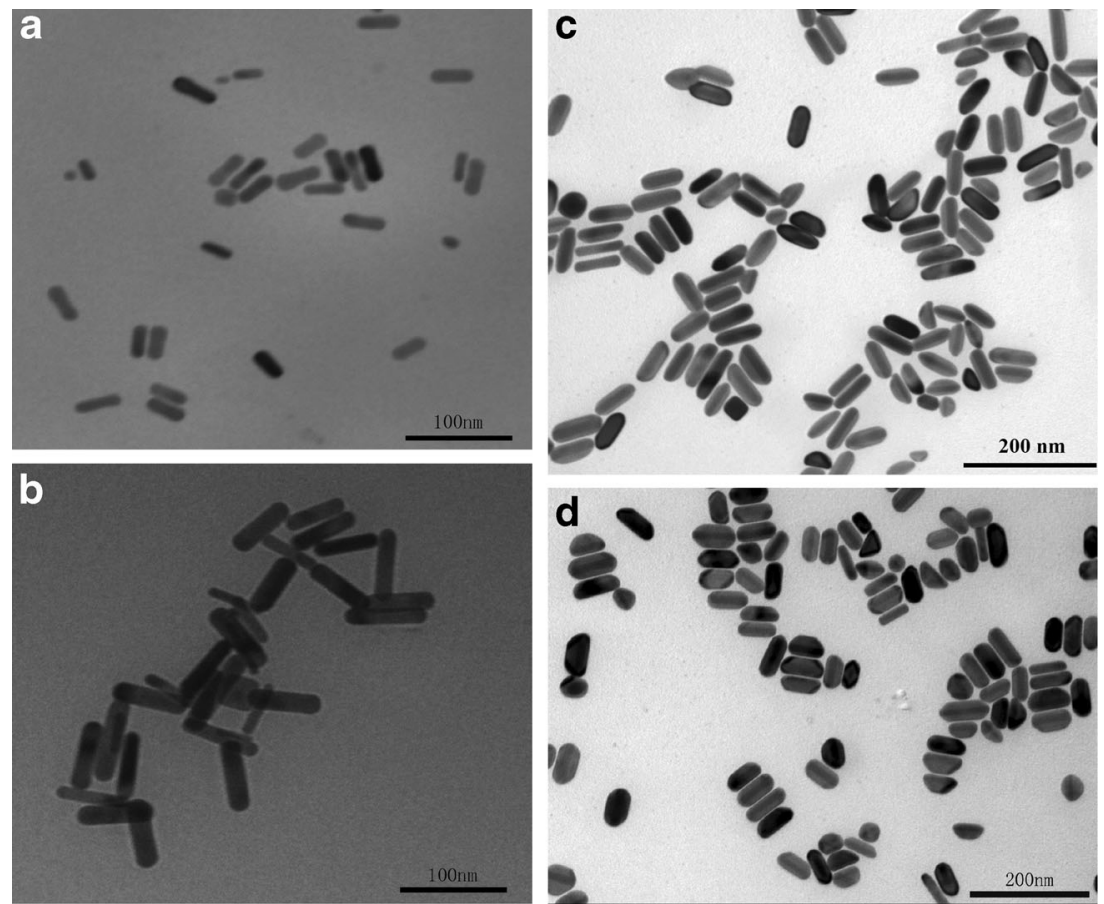

of 4.8 (with an average length of about $62 \mathrm{~nm}$ and width of about $13 \mathrm{~nm}$ ) were obtained.

\section{The synthesis of Au-Ag core-shell nanorods}

Au nanorods with Ag coating were prepared by the method in previous literature with some minor modification $[3,16,29$, 30]. In summary, $45 \mathrm{~mL}$ prepared $\mathrm{Au}$ nanorods were centrifuged for $20 \mathrm{~min}$ at $10,000 \mathrm{rpm}$, then the supernatant was carefully removed and the precipitate was redispersed into the same volume of $0.08 \mathrm{M}$ CTAB. One minute of the ultrasonic solution is needed. Afterwards, the solution is divided into nine portions. For each portion, $\mathrm{AgNO}_{3}(0.01 \mathrm{M}), \mathrm{AA}(0.1 \mathrm{M}$, $0.4 \mathrm{~mL})$, and $\mathrm{NaOH}(0.1 \mathrm{M}, 1 \mathrm{~mL})$ was added in that order. By adding the volume of $\mathrm{AgNO}_{3}$ from 0.1 to $0.9 \mathrm{~mL}$, we obtained $\mathrm{Au}-\mathrm{Ag}$ core-shell nanorods with different $\mathrm{Ag}$ shell thickness. The solution was then kept at $65^{\circ} \mathrm{C}$ for $4 \mathrm{~h}$. The typical TEM images of the synthesized $\mathrm{Au}-\mathrm{Ag}$ core-shell nanorods with thin Ag coating and thick Ag coating are compared. As can be observed in Fig. 5c, when the volume of $\mathrm{AgNO}_{3}$ is $0.5 \mathrm{~mL}$, the $\mathrm{Ag}$ shell at the side facet is thin, whose average thickness is about $5 \mathrm{~nm}$. And thicker Ag shell at the side facet of about $10 \mathrm{~nm}$ could be observed when the volume of $\mathrm{AgNO}_{3}$ reaches $0.8 \mathrm{~mL}$ as shown in Fig. 5 d.

Figure 6a depicts the absorption spectra of $\mathrm{Au}-\mathrm{Ag}$ core-shell nanorods with longer Au nanorods core. As can be seen in Fig. 6a, for longer gold nanorods, as the volume of $\mathrm{AgNO}_{3}$ is increased, peak 1 slightly red shifts with intensity increasing quickly, while peak 3 blue shifts greatly with intensity increasing slowly. At the same time, peak 2 attenuated and then disappeared. The intensity difference between peaks 1 and 3 becomes smaller and smaller with the continuous increase of $\mathrm{AgNO}_{3}$. We can obtain two intense plasmonic absorption peaks with equal intensity when the volume of $\mathrm{AgNO}_{3}$ reaches $0.85 \mathrm{~mL}$, as shown in Fig. 6a. For shorter gold nanorods, two intense plasmonic absorption peaks with equal intensity appeared when the volume of $\mathrm{AgNO}_{3}$ reaches $0.4 \mathrm{~mL}$ as shown in Fig. 6b. As the volume of $\mathrm{AgNO}_{3}$ is further increased, peak 1 exceeds peak 3 and leaves only one peak at about $450 \mathrm{~nm}$ at last. As a matter of fact, longer and shorter gold nanorods represent aspect ratios of large and small, the volume of $\mathrm{AgNO}_{3}$ corresponds to the thickness of silver coating. The silver coating gets thicker as the amount of $\mathrm{AgNO}_{3}$ is increased. A thin silver layer was developed at first, as shown in line A of Fig. 6b. Owing to its nonhomogeneous coating, the increase of the side of the nanorod's thickness is faster than the end as shown in line B of Fig. 6b. It is obvious that its side facet of silver coating is thicker than line A of Fig. 6b. At last, the nanorods turned to nearly spherical structure as shown in line $\mathrm{C}$ of Fig. 6b. Figure $6 \mathrm{c}, \mathrm{d}$ depicts the dependences of the plasmon peak wavelengths and absorption intensities on the volume of the $\mathrm{AgNO}_{3}$ solution. Because the $\mathrm{Ag}$ shell thickness is controlled by the amount of $\mathrm{AgNO}_{3}$, the amount of $\mathrm{AgNO}_{3}$ corresponds to the Ag shell thickness, and the $\mathrm{Ag}$ shell thickness is increased as the volume of the $\mathrm{AgNO}_{3}$ solution is increased. Increasing the silver coating results in the 

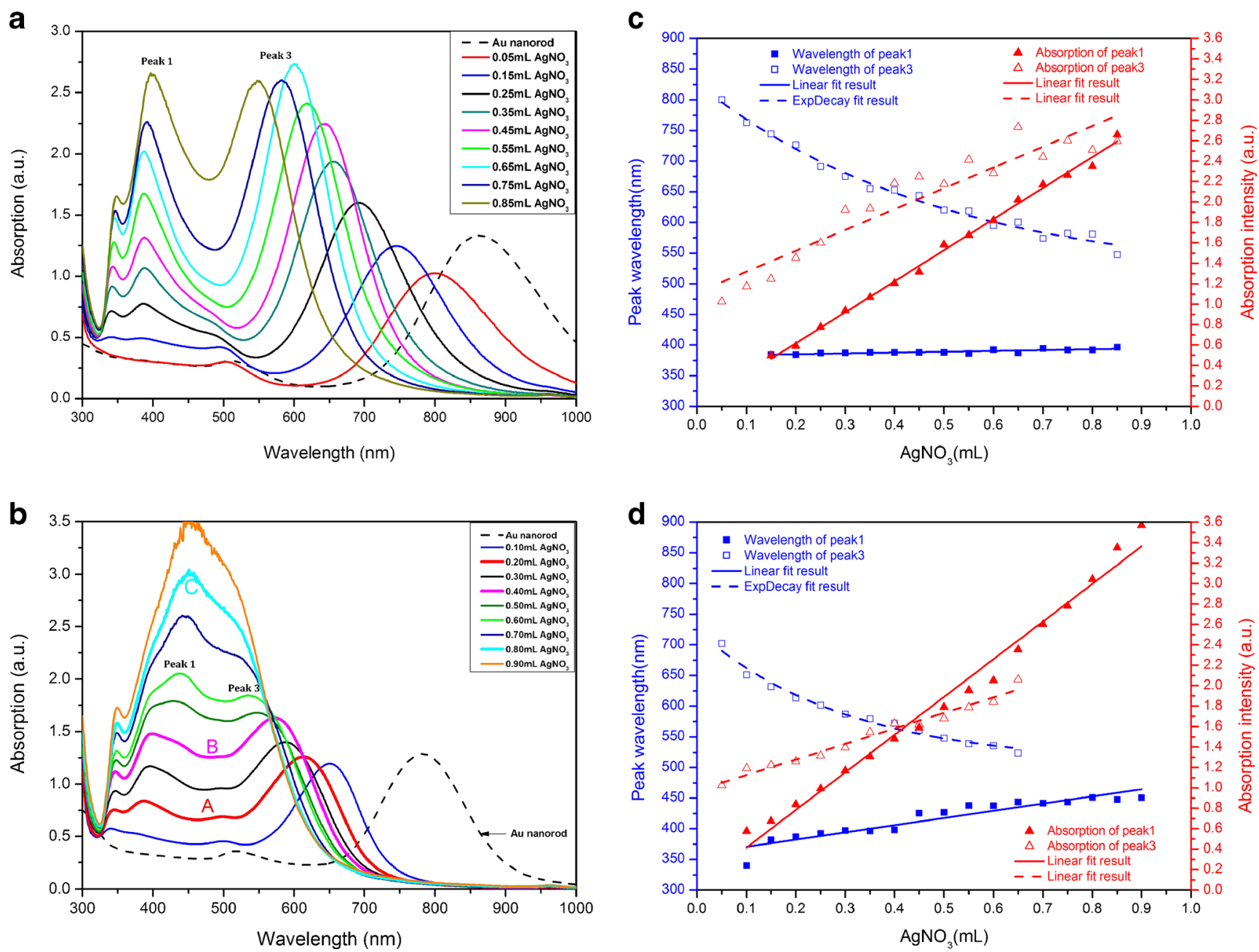

Fig. 6 Absorption spectra of $\mathrm{Au}$ nanorod samples and $\mathrm{Au}-\mathrm{Ag}$ core-shell nanorod samples with different volume of the $0.01 \mathrm{M} \mathrm{AgNO}_{3}$ solution. a The $\mathrm{Au}$ nanorods have large aspect ratio and the $\mathrm{AgNO}_{3}$ volume is increased from 0.05 to $0.85 \mathrm{~mL}$ with an increment of $0.1 \mathrm{~mL}$. b The $\mathrm{Au}$ nanorods have small aspect ratio and the $\mathrm{AgNO}_{3}$ volume is increased

from 0.1 to $0.9 \mathrm{~mL}$ with an increment of $0.1 \mathrm{~mL}$. Dependences of the plasmon peak wavelengths and absorption intensities on the volume of the $\mathrm{AgNO}_{3}$ solution, the Au nanorods have a $\mathbf{c}$ large aspect ratio and $\mathbf{d}$ small aspect ratio

absorption intensity of both peaks 1 and 3 , getting intense linearly. The linear relation could also be found for the silver coating-dependent plasmon shifting of peak 1 . However, the peak 3 blue shift exponentially as the silver coating is increased. Furthermore, one can find the silver coating-dependent intensity increasing of peak 1 is more intense as the gold nanorods are short. Therefore, two absorption peaks with equal intensity could be obtained with thinner silver coating. These experimental observations are in good agreement with our theoretical calculation above.

\section{Conclusions}

In conclusion, nonhomogeneous $\mathrm{Ag}$ coating of the $\mathrm{Au}$ nanorod and aspect ratio of $\mathrm{Au}$ nanorod core strongly affect

the intensity change and wavelength shift of the plasmonic absorption. Theoretical calculations show that, when the transverse $\mathrm{Ag}$ coating is faster, the $\mathrm{Au}-\mathrm{Ag}$ core-shell nanorod could present two intense longitudinal plasmonic absorption peaks with equal intensity and small wavelength gap as the $\mathrm{Ag}$ shell thickness has a critical value. When the longitudinal Ag coating is faster, the intensity increasing of longitudinal peak corresponding to outer $\mathrm{Ag}$ surface is always weaker than the longitudinal peak corresponding to $\mathrm{Au}-\mathrm{Ag}$ interface. The critical value of Ag shell thickness could be decreased when $\mathrm{Au}$ nanorod is short, at the same time the transverse peak corresponding to $\mathrm{Au}-\mathrm{Ag}$ interface could be enhanced. It is worth noting that the two equal intensity of absorption peaks exhibit $\mathrm{Au}-\mathrm{Ag}$ core-shell nanorod obtained from the nonhomogeneous silver coating may have great potential for many biological and chemical applications such as design and fabrication of dual-channel SPR sensor. 
Acknowledgments This work was supported by the Program for New Century Excellent Talents in University under grant no. NCET-10-0688, the Fundamental Research Funds for the Central Universities under grant no. 2011jdgz17, and the National Natural Science Foundation of China under grant nos. 11174232,61178075 , and 81101122 .

Open Access This article is distributed under the terms of the Creative Commons Attribution License which permits any use, distribution, and reproduction in any medium, provided the original author(s) and the source are credited.

\section{References}

1. Moskovits M, Srnová-Śloufová I, Vlčkova B (2002) Bimetallic Ag$\mathrm{Au}$ nanoparticles: extracting meaningful optical constants from the surface-plasmon extinction spectrum. J Chem Phys 116:10435-10446

2. Xu HX (2005) Multilayered metal core-shell nanostructures for inducing a large and tunable local optical field. Phys Rev B 72: 0734051-0734055

3. Jiang R, Chen H, Shao L, Li Q, Wang J (2012) Unraveling the evolution and nature of the plasmons in (Au core) $-(\mathrm{Ag}$ shell) nanorods. Adv Mater 24:OP200-OP207

4. Zhang Q, Moran CH, Xia XH, Rycenga M, Li NX, Xia YN (2012) Synthesis of Ag nanobars in the presence of single-crystal seeds and a bromide compound, and their surface-enhanced Raman scattering (SERS) properties. Langmuir 28:9047-9054

5. Li Y, Jing C, Zhang L, Long YT (2012) Resonance scattering particles as biological nanosensors in vitro and in vivo. Chem Soc Rev 41:632-642

6. Wu LP, Li YF, Huang CZ, Zhang Q (2006) Visual detection of Sudan dyes based on the plasmon resonance light scattering signals of silver nanoparticles. Anal Chem 78:5570-5577

7. Tao AR, Habas S, Yang PD (2008) Shape control of colloidal metal nanocrystals. Small 4:310-325

8. Chen HJ, Shao L, Woo KC, Ming T, Lin HQ, Wang JF (2009) Shapedependent refractive index sensitivities of gold nanocrystals with the same plasmon resonance wavelength. J Phys Chem C 113:17691-17697

9. Cardinal MF, Rodríguez-González B, Alvarez-Puebla RA, PérezJuste J, Liz-Marzán LM (2010) Modulation of localized surface plasmons and SERS response in gold dumbbells through silver coating. J Phys Chem C 114:10417-10423

10. Tsuji M, Miyamae N, Lim S, Kimura K, Zhang X, Hikino S, Nishio M (2006) Crystal structures and growth mechanisms of Au@Ag core-shell nanoparticles prepared by the microwave-polyol method. Cryst Growth Des 6:1801-1807

11. Xue C, Millstone JE, Li S, Mirkin CA (2007) Plasmon-driven synthesis of triangular core-shell nanoprisms from gold seeds. Angew Chem Int Ed 46:8436-8439

12. Duan J, Park K, MacCuspie RI, Vaia RA, Pachter R (2009) Optical properties of rodlike metallic nanostructures: insight from theory and experiment. J Phys Chem C 113:15524-15532
13. Tsuji M, Matsuo R, Jiang P, Miyamae N, Ueyama D, Nishio M, Hikino S, Kumagae H, Kamarudin KSN, Tang XL (2008) Shapedependent evolution of $\mathrm{Au} @ \mathrm{Ag}$ core-shell nanocrystals by PVPassisted $N, N$-dimethylformamide reduction. Cryst Growth Des 8: $2528-2536$

14. Gong JX, Zhou F, Li ZY, Tang ZY (2012) Synthesis of Au@Ag core-shell nanocubes containing varying shaped cores and their localized surface plasmon resonances. Langmuir 28:8959-8964

15. Hong SC, Choi YJ, Park SH (2011) Shape control of Ag shell growth on Au nanodisks. Chem Mater 23:5375-5378

16. Ma YY, Li WY, Cho EC, Li ZY, Yu TY, Zeng J, Xie ZX, Xia YN (2010) Au@Ag core-shell nanocubes with finely tuned and wellcontrolled sizes, shell thicknesses, and optical properties. ACS Nano 4:6725-6734

17. Deng JJ, Du J, Wang Ye TYF, Di JW (2011) Synthesis of ultrathin silver shell on gold core for reducing substrate effect of LSPR sensor. Electrochem Commun 13:1517-1520

18. Lee YH, Chen H, Xu QH, Wang J (2011) Refractive index sensitivities of noble metal nanocrystals: the effects of multipolar plasmon resonances and the metal type. J Phys Chem C 115:7997-8004

19. Zhu J (2009) Surface plasmon resonance from bimetallic interface in $\mathrm{Au}-\mathrm{Ag}$ core-shell structure nanowires. Nanoscale Res Lett 4:977981

20. Yu K, You GJ, Polavarapu L, Xu QX (2011) Bimetallic Au/ Ag core-shell nanorods studied by ultrafast transient absorption spectroscopy under selective excitation. J Phys Chem C 115:14000-14005

21. Liu MZ, Guyot-Sionnest P (2004) Synthesis and optical characterization of $\mathrm{Au} / \mathrm{Ag}$ core/shell nanorods. Phys Chem B 108:5882-5888

22. Perenboom JAAJ, Wyder P, Meier F (1981) Electronic properties of small metallic particles. Phys Rep 78:173-292

23. Liu MZ, Guyot-Sionnest P (2006) Preparation and optical properties of silver chalcogenide coated gold nanorods. J Mater Chem 16:39423945

24. Yi DK (2011) A study of optothermal and cytotoxic properties of silica coated Au nanorods. Mater Lett 65:2319-2321

25. Huang H, Liu X, Zeng Y, Yu X, Liao B, Yi P, Chu PK (2009) Optical and biological sensing capabilities of $\mathrm{Au} 2 \mathrm{~S} / \mathrm{AuAgS}$ coated gold nanorods. Biomaterials 30:5622-5630

26. Marinakos SM, Chen S, Chilkoti A (2007) Plasmonic detection of a model analyte in serum by a gold nanorod sensor. Anal Chem 79: $5278-5283$

27. Chakravadhanula VSK, Elbahri M, Schürmann U, Takele H, Greve H, Zaporojtchenko V, Faupel F (2008) Equal intensity double plasmon resonance of bimetallic quasi-nanocomposites based on sandwich geometry. Nanotechnology 19:225302-225306

28. Sau TK, Murphy CJ (2004) Seeded high yield synthesis of short Au nanorods in aqueous solution. Langmuir 20:6414-6120

29. Fu Q, Zhang DG, Yi MF, Wang XX, Chen YK, Wang P, Ming H (2012) Effect of shell thickness on a Au-Ag core-shell nanorodsbased plasmonic nano-sensor. J Opt 14:085001-085005

30. Zhu J, Zhang F, Li JJ, Zhao JW (2013) Optimization of the refractive index plasmonic sensing of gold nanorods by non-uniform silver coating. Sensor Actuat B-Chem 183:556-564 